\title{
Gar nicht so selten
}

Patienten mit ambulant erworbener Pneumonie können fieberfrei, aber trotzdem bakteriämisch sein, wie die deutsche CAPNETZ-Studie belegt. Dessen sollte man sich auch in Hinblick auf das Sepsis-Risiko bewusst sein.

Im ambulanten Bereich erworbene Lungenentzündungen werden immer noch unterschätzt, erklärt Dr. Jessica Rademacher von der Medizinischen Hochschule Hannover. Dabei zähle die CAP (community acquired pneumonia) in Deutschland zu den häufigsten infektionsbedingten Todesursachen. Studien haben gezeigt, dass jährlich mehr Menschen wegen einer ambulant erworbenen Pneumonie stationär behandelt werden als wegen Herzinfarkt oder Schlaganfall; die Klinikmortalität liege zwischen zwölf und $14 \%$.

Die Pneumonie ist auch die häufigste Ursache für eine ambulant erworbene Sepsis betont Rademacher. Daher sei es „extrem wichtig", einer eventuell vorliegenden Bakteriämie so rasch wie möglich auf die Spur zu kommen.

Als Goldstandard zur Diagnostik der Bakteriämie gilt die Blutkultur. Deren Rolle bei CAP-Patienten ist jedoch nach wie vor umstritten. Tatsächlich scheint der Nutzen im ambulanten Bereich relativ gering zu sein. Wie die kürzlich publizierte CAPNETZ-Studie gezeigt hat, lag die Rate positiver Blutkulturen bei CAP-Patienten insgesamt deutlich unter zehn Prozent (292 von 4349 getesteten Teilnehmern). In der Studie blieben letztlich nur drei Patienten mit einer Bakteriämie ambulant. Von diesen war zum Studienende niemand gestorben.

\section{Ein Drittel der bakteriämischen Patienten ohne Fieber!}

Überraschend war jedoch der hohe Anteil an bakteriämischen Patienten ohne Fieber. Dieser betrug laut Rademacher knapp ein Drittel der Teilnehmer mit positiver Blutkultur. Gerade diese Patienten waren offenbar besonders gefährdet: So war die Mortalitätsrate bei den afebrilen bakteriämischen Patienten mit 9,9\% mehr als doppelt so hoch als in der Gruppe mit febriler Bakteriämie (3,7\%).

Prädiktoren für eine afebrile Bakteriämie waren in der CAPNETZ-Studie

- ein positiver PneumokokkenAntigen-Test,

- ein CRP-Wert > $200 \mathrm{mg} / \mathrm{l}$ und

- ein Harnstoffwert $\geq 30 \mathrm{mg} / \mathrm{dl}$.

Für die Praxis bedeute dies, so Rademacher: Während die Blutkultur bei einer stationär zu behandelnden Pneumonie unbedingt dazu gehört, scheint sie im ambulanten Bereich verzichtbar zu sein. Neben dem klinischen Bild sollten etablierte Scores wie der CRB-65 zur Einschätzung des Letalitätsrisikos eingesetzt werden - dessen Verlässlichkeit wurde in einer neueren Metaanalyse bestätigt. Dieser sollte allerdings noch um die Parameter "Hypoxämie" und "potenziell instabile Komorbiditäten" sowie "chronische Bettlägerigkeit" ergänzt werden.

\section{Ältere Patienten häufig ohne klassische Symptome}

Fehlendes Fieber sei, so Rademacher, grundsätzlich „kein Grund, eine Blutkultur nicht durchzuführen“. Letztere ermögliche zuweilen erst die leitliniengerechte Behandlung: So konnte bei immerhin zehn afebrilen Patienten eine Staphylococcus-aureus-Bakteri-

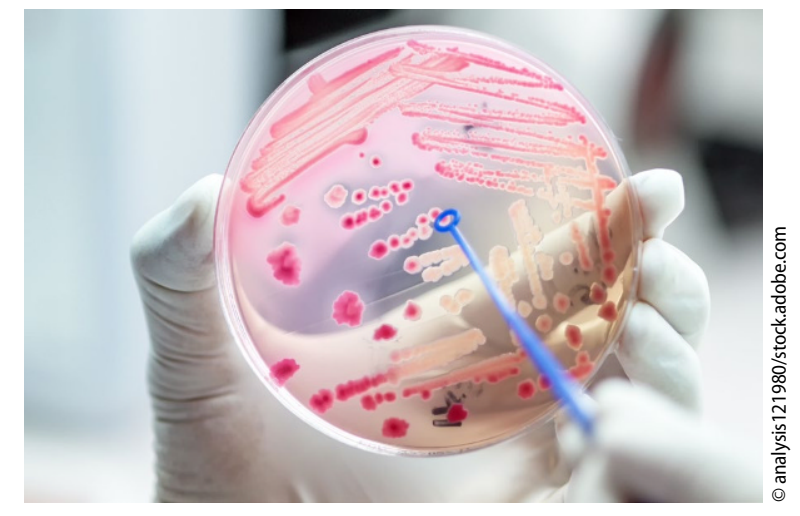

ämie diagnostiziert werden. Der häufigste Erreger bei den Patienten ohne Fieber war S. pneumoniae, gefolgt von E. coli.

Laut den Autoren der CAPNETZStudie wird Fieber oft als Trigger für das Anfertigen einer Blutkultur angesehen. Dies lasse jedoch außer Acht, dass vor allem ältere Patienten häufig eben nicht die klassischen Symptome einer CAP zeigen. In der Studie waren die afebrilen bakteriämischen Patienten im Mittel 70 Jahre alt.

\section{Elke Oberhofer}

Hinweis des Verlags. Der Verlag bleibt in Hinblick auf geografische Zuordnungen und Gebietsbezeichnungen in veröffentlichten Karten und Institutsadressen neutral.

Wien klin Mag 2020 · 23:297 https://doi.org/10.1007/s00740-02000377-7

(c) Springer-Verlag GmbH Austria, ein Teil von Springer Nature 2020
Quelle: Rademacher J. Pneumonie. Infektio-Update, 4./5. September 2020, online 